\title{
Glycolysis Is Required for LPS-Induced Activation and Adhesion of Human CD14 ${ }^{+}$CD16 ${ }^{-}$ Monocytes
}

OPEN ACCESS

Edited by:

Kate E. Lawlor,

Hudson Institute of Medical

Research, Australia

Reviewed by:

Nicole K. Campbell,

Hudson Institute of Medical

Research, Australia

Evanna Mills,

Dana-Farber Cancer Institute, United States

Kristiaan Wouters,

Maastricht University, Netherlands Laurent Yvan-Charvet,

Institut National de la Santé et de la

Recherche Médicale

(INSERM), France

${ }^{*}$ Correspondence:

Man K. S. Lee

mankitsam.lee@baker.edu.au

Andrew J. Murphy

Andrew.murphy@baker.edu.au

tThese authors have contributed equally to this work

Specialty section:

This article was submitted to

Molecular Innate Immunity,

a section of the journal

Frontiers in Immunology

Received: 08 April 2019

Accepted: 14 August 2019

Published: 06 September 2019

Citation:

Lee MKS, Al-Sharea A, Shihata WA,

Bertuzzo Veiga $C$, Cooney $O D$,

Fleetwood AJ, Flynn MC, Claeson E,

Palmer CS, Lancaster Gl,

Henstridge DC, Hamilton JA and

Murphy AJ (2019) Glycolysis is

Required for LPS-Induced Activation

and Adhesion of Human

$\mathrm{CD}^{+} 4^{+} \mathrm{CD} 16^{-}$Monocytes.

Front. Immunol. 10:2054

doi: 10.3389/fimmu.2019.02054

\begin{abstract}
Man K. S. Lee ${ }^{1,2 * t}$, Annas Al-Sharea ${ }^{1,2 t}$, Waled A. Shihata ${ }^{1}$, Camilla Bertuzzo Veiga ${ }^{1}$, Olivia D. Cooney ${ }^{1}$, Andrew J. Fleetwood ${ }^{3,4}$, Michelle C. Flynn ${ }^{1}$, Ellen Claeson ${ }^{5}$, Clovis S. Palmer ${ }^{6}$, Graeme I. Lancaster ${ }^{1}$, Darren C. Henstridge ${ }^{1}$, John A. Hamilton ${ }^{3,4}$ and Andrew J. Murphy ${ }^{1 *}$

${ }^{1}$ Division of Immunometabolism, Baker Heart and Diabetes Institute, Melbourne, VIC, Australia, ${ }^{2}$ Department of Diabetes, Monash University, Melbourne, VIC, Australia, ${ }^{3}$ Department of Medicine, The Royal Melbourne Hospital, Parkville, VIC, Australia, ${ }^{4}$ Australian Institute of Musculoskeletal Science, University of Melbourne and Western Health, St. Albans, VIC, Australia, ${ }^{5}$ Faculty of Medicine and Health Sciences, Linköping University, Linköping, Sweden, ${ }^{6}$ Department of Infectious Disease, Burnet Institute, Melbourne, VIC, Australia
\end{abstract}

Monocytes in humans consist of 3 subsets; $\mathrm{CD}_{14}^{+} \mathrm{CD}^{-} 6^{-}$(classical), $\mathrm{CD}_{14}{ }^{+} \mathrm{CD} 16{ }^{+}$(intermediate) and $\mathrm{CD}^{2} 4^{\mathrm{dim}} \mathrm{CD} 16^{+}$(non-classical), which exhibit distinct and heterogeneous responses to activation. During acute inflammation CD14 ${ }^{+} \mathrm{CD}_{16}{ }^{-}$monocytes are significantly elevated and migrate to the sites of injury via the adhesion cascade. The field of immunometabolism has begun to elucidate the importance of the engagement of specific metabolic pathways in immune cell function. Yet, little is known about monocyte metabolism and the role of metabolism in mediating monocyte activation and adherence to vessels. Accordingly, we aimed to determine whether manipulating the metabolism of $\mathrm{CD} 14^{+} \mathrm{CD} 16^{-}$monocytes alters their ability to become activated and adhere. We discovered that LPS stimulation increased the rate of glycolysis in human $\mathrm{CD} 14^{+} \mathrm{CD}_{16}{ }^{-}$monocytes. Inhibition of glycolysis with 2-deoxy-D-glucose blunted LPS-induced activation and adhesion of monocytes. Mechanistically, we found that increased glycolysis was regulated by mTOR-induced glucose transporter (GLUT)-1. Furthermore, enhanced glycolysis increased accumulation of reactive oxygen species (ROS) and activation of p38 MAPK, which lead to activation and adhesion of monocytes. These findings reveal that glycolytic metabolism is critical for the activation of $\mathrm{CD}_{14}{ }^{+} \mathrm{CD} 16^{-}$monocytes and contributes to our understanding of the interplay between metabolic substrate preference and immune cell function.

Keywords: glycolysis, monocytes, inflammation, metabolism, adhesion

\section{INTRODUCTION}

Innate immune cells such as monocytes play an essential role during inflammation. Monocytes emerge from the bone marrow or spleen into the blood when inflammatory cues are released from sites of injury and ultimately migrate from the blood into the inflamed tissue. This is commonly referred to as the leukocyte adhesion cascade (1). This is a stepwise process initially involving the activation of monocytes, allowing them to roll and tether roll and tether 
along the activated endothelium followed by firm adhesion and extravasation into inflamed sites. These events are necessary for the survival of the host, as inflammation is important for clearing invading pathogens and repairing damaged tissues (2). On the other hand, excessive inflammation can lead to detrimental effects, resulting in further damage to tissues, and the development of chronic inflammatory diseases such as atherosclerosis and other auto-inflammatory disorders (3-5). Therefore, understanding the underlying mechanisms that regulate monocyte adhesion can provide information on how to manipulate the cell's ability to adhere during acute or chronic inflammation.

It has become increasingly appreciated that the metabolic status of a cell can dictate its functional phenotype. We and others have shown that stimulating cells with inflammatory stimuli switches the energy profile of macrophages and $\mathrm{T}$ cells, i.e., from using mitochondrial oxidative phosphorylation (OXPHOS) to glycolysis (6-8). Changes in the cellular substrates and metabolic preferences under resting and activated states has sparked investigations into further understanding immune cell metabolism (i.e., immunometabolism) in relation to their function. It has become increasingly evident that manipulating metabolic pathways influences the development and function of cells. For example, inhibiting glycolysis in LPS-stimulated bone marrow-derived macrophages (BMDMs), prevents the release of the inflammatory cytokine interleukin (IL)-1 $\beta$ (9).

In humans, blood monocytes consist of 3 functionally distinct subsets, $\mathrm{CD}_{14}{ }^{+} \mathrm{CD} 16^{-}$(classical), $\mathrm{CD} 14^{+} \mathrm{CD} 16^{+}$ (intermediate), and $\mathrm{CD} 14^{\mathrm{dim}} \mathrm{CD}^{-} 6^{-}$(non-classical) (10). $\mathrm{CD} 14^{+} \mathrm{CD} 16^{-}$monocytes represent $85 \%$ of the circulating monocyte population whereas the other two subsets each make up $\sim 5-8 \%$ of the population. In mice, however, monocytes consist of only two subsets, Ly6-C ${ }^{\text {hi }}$ and Ly6-C $C^{\text {lo }}$ Ly6-C ${ }^{\text {hi }}$ monocytes are considered to be inflammatory and have been likened to the $\mathrm{CD} 14^{+} \mathrm{CD} 16^{-}$monocyte population in humans. During acute inflammation, such as a bacterial infection, these are the subsets that respond first. Therefore, in this study we aimed to investigate how human $\mathrm{CD} 14^{+} \mathrm{CD} 16^{-}$ monocytes metabolically respond to inflammatory stimuli such as lipopolysaccharide (LPS) and how manipulating monocyte metabolism could alter their functional responses, particularly in relation to their activation and ability to undergo firm adhesion.

\section{MATERIALS AND METHODS}

\section{Isolation of Peripheral Blood Mononuclear Cells From Human Buffy Coats}

Peripheral blood mononuclear cells (PBMC) were isolated from buffy coats of healthy volunteers supplied by the Australian Red Cross Blood Service via density-centrifugation using FicollPaque solution (density $=1.77$ ). Ethics was obtained through the Alfred Hospital human ethics committee.

\section{CD14 ${ }^{+}$CD16- Monocyte Isolation From PBMCs}

PBMCs were resuspended using PBS without $\mathrm{Ca}^{2+}$ and $\mathrm{Mg}^{2+}$ containing $2 \mathrm{mM}$ EDTA and 5\% FBS (FACS buffer) and labeled with a cocktail of fluorescent markers (1:400) consisting of Lin (PE-CD2, PE-CD15, PE-CD56, PE-Nkp46, PE-19), APC-HLA-DR, PB-CD14, PE/Cy7-CD16. After $30 \mathrm{~min}$ of incubation on ice, they were then washed and $\mathrm{Lin}^{-} \mathrm{HLA}-$ $\mathrm{DR}^{+} \mathrm{CD} 14^{+} \mathrm{CD}^{-} 6^{-}$monocytes were collected via FACS using the $\mathrm{BD}$ Aria 1 (Biosciences) at the AMREP Flow cytometry core facility.

\section{Stimulation/Inhibition of Cells}

PBMCs (for flow cytometry assays) and isolated CD $14^{+} \mathrm{CD} 16^{-}$ monocytes were stimulated with lipopolysaccharides (LPS) $(100 \mathrm{ng} / \mathrm{ml})$ for $1 \mathrm{~h}$ in the presence or absence of $1 \mathrm{~h}$ of metabolic inhibitor pre-treatment; glycolysis inhibitor: 2-DeoxyD Glucose (2DG) (5 mM), mechanistic target of rapamycin (mTOR) inhibitor: rapamycin $(20 \mathrm{nM})$, reactive oxygen species (ROS) inhibitor: NAC (1 mM), p38 MAPK inhibitor: SB-203580 $(5 \mathrm{nM})$ and mitochondrial ROS scavenger: MitoQ (100 nM).

\section{Flow Cytometry}

PBMCs were resuspended in $200 \mu \mathrm{l}$ of FACs buffer. A cocktail of the fluorophores $(1: 400)$ were added to stain for the different monocyte subsets. These consisted of Lin (PE-CD2, PECD15, PE-CD56, PE-Nkp46, PE-19), APC-HLA-DR, PBCD14, PE/Cy7-CD16. To measure CD11b levels or GLUT1 expression, FITC- CD11b (1:400), and FITC-GLUT-1 was also added, respectively. After incubating on ice for $30 \mathrm{~min}$, they were then washed and transferred into FACS tubes. In order to measure metabolism of cells via flow cytometry, $10 \mathrm{nM}$ MitoTracker Deep Red, $4 \mu \mathrm{M}$ 2-NBDG, $5 \mu \mathrm{M}$ MitoSOX, and $10 \mu \mathrm{M}$ H2DCFDA were stained in RPMI 1640 and incubated in $37^{\circ} \mathrm{C}$ for $20 \mathrm{~min}$ before they were washed and transferred into FACS tubes. Cells were immediately run on the BD LSRII Fortessa (BD Biosciences). 100,000 cells were collected for analysis. Unstained and single stained controls were used to set up voltages to compensate for spectral overlap. Flow cytometry data were quantified using FlowJo vX0.7 (FlowJo LCC) software.

\section{Seahorse Bioanalyser Assay}

Monocytes were pre-treated with inhibitors and seeded at 100,000 cells/well in the $\mathrm{XF}^{\mathrm{e}} 96$ well cell culture microplate (Agilent Technologies). The microplate was spun at 1,000 RPM for $5 \mathrm{~min}$ at $4^{\circ} \mathrm{C}$; acc $=5$, dec $=0$ to obtain a monolayer of monocytes in each well. The supernatant was discarded and $175 \mu$ l of seahorse media [XF based minimal DMEM (Agilent Technologies) supplemented with $5.5 \mathrm{mM}$ Glucose Solution (Gibco), $1 \mathrm{mM}$ Sodium Pyruvate (Gibco) and $2 \mathrm{mM} \mathrm{L-Glutamine}$ (Gibco)], containing the same concentrations of inhibitors, were carefully added so as not to disturb the cell layer. The $\mathrm{XF}^{\mathrm{e}} 96$ well cell culture microplate was incubated at $37^{\circ} \mathrm{C}$ in a non$\mathrm{CO}_{2}$ incubator for at least $30 \mathrm{~min}$. The assay cartridge was hydrated overnight with $200 \mu \mathrm{l}$ of XF Calibrant Media (Agilent Technologies) at $37^{\circ} \mathrm{C}$ (in non- $\mathrm{CO}_{2}$ incubator) before LPS was suspended in $25 \mu \mathrm{l}$ of seahorse media and was added to Port A of the assay cartridge at a concentration of $100 \mathrm{ng} / \mathrm{ml}$. Basal extracellular acidification rate (ECAR) was measured for $4 \times$ 6.5 min cycles. LPS was automatically injected into the $\mathrm{XF}^{\mathrm{e}}$ cell culture plate after the 4 th cycle and ECAR readings were recorded for $1 \mathrm{~h}$ post-LPS injection. 


\section{Vessel Chamber Adhesion Assay}

Aortic vessels were isolated from C57BL/6 mice and stimulated in Krebs buffer with bovine serum albumin (BSA) $(1: 1,000$ $\mathrm{w} / \mathrm{v}$ ) and TNF- $\alpha$ for $4 \mathrm{~h}$ at $37^{\circ} \mathrm{C}$. TNF- $\alpha$ activated vessels were mounted onto the cannulas and Krebs solution warmed at $37^{\circ} \mathrm{C}$ was used to flood the vessel chamber to mimic in-vivo conditions. Monocytes were re-suspended in $6 \mathrm{ml}$ of RPMI at the concentration of $1 \times 10^{6}$ cells $/ \mathrm{ml} .1 \mathrm{mM}$ of Vybrant Dil (Invitrogen) was added for $10 \mathrm{~min}$ in dark conditions, to fluorescently label the cells. Cell solutions were transferred into a terafusion syringe pump (Teruma) which was used to direct the movement of the cells through the aortic vessel at a rate of $7.1 \mathrm{ml}$ per hour. Images of adhered monocytes were taken using the Zesiss Discover V.20 Fluorescence Microscope (Carl Zeiss MicroImaging) mounted on a Hammastsu HD Camera (Hamamatsu $^{\circledR}$ ) at $0,2.5,5,7.5$, and $10 \mathrm{~min}$. Data was then quantified by calculating the number of stationary fluorescent dots per field of view (FOV).

\section{F-Actin Assay}

Eight well chamber slides (Lab-Tek) were pre-coated with 200 $\mu \mathrm{l} /$ well of fibrinogen $(100 \mu \mathrm{g} / \mathrm{ml})$ and incubated overnight at $4^{\circ} \mathrm{C}$. The next day, each well was washed twice with PBS without $\mathrm{Mg}^{2+}$ and $\mathrm{Ca}^{2+}$ to get rid of non-adhered fibrinogen via aspiration. Two-hundred microliter of 3\% BSA were then added and incubated at room temperature for 15 minutes before washing again with PBS twice. Two-hundred microliter of monocytes (at $1 \times 10^{6} / \mathrm{ml}$ in RPMI 1640 media) were then added into each well and stimulated with $100 \mathrm{ng} / \mathrm{ml} \mathrm{LPS} \mathrm{for} 1 \mathrm{~h}$ at $37^{\circ} \mathrm{C}$. Cells were then washed with PBS twice to remove unbound monocytes. Two-hundred microliter of $4 \%$ para-formaldehyde (PFA) was then added for $15 \mathrm{~min}$ at room temperature to fix adhered monocytes onto slides. Again, PBS was used to wash off the PFA before permeabilising cells with $0.1 \%$ Triton X-100 for $10 \mathrm{~min}$ at room temperature. After washing cells with PBS, 200 $\mu \mathrm{l}$ of PBS containing $10 \%$ FBS was used to block any non-specific binding for $15 \mathrm{~min}$ at room temperature. Cells were washed again before staining with fluorescent markers of F-actin (33 nM phalloidin) and nucleus ( $1 \mathrm{ng} / \mathrm{ml} \mathrm{4}$, 6-diamidino-2-phenylindole (DAPI) for $20 \mathrm{~min}$ in the dark at room temperature. Cells were then washed twice with PBS and the gasket were removed. slides were finally allowed to completely dry before mounting on No. 2 glass coverslips (Menzel) using Dako fluorescence mounting media. Imaging was performed through the monash micro imaging core, on a Nikon A1r confocal microscope using NISelements software (Nikon) at 60X magnification. To quantify F-actin staining, the fluorescence intensity of phalloidin stain per cell, normalized to cell size, was measured using Image J. Moreover, cells were counted individually, using the Image J count function, to quantify the number of adherent monocytes.

\section{Western Blot}

Protein samples were isolated from lysed Monocytes. A 10\% SDS-PAGE gel was used to separate the protein samples which were subsequently transferred onto a nitrocellulose membrane. Five percent fat-free skim milk in tris-buffered saline with tween (TBST) was used to block the membrane for nonspecific binding and washed with before the addition of various primary antibodies $(1: 1,000)$ consisting of p-mTOR (Santa Cruz), p-ERK1/2 (Cell Signaling Technologies), $\beta$-actin (Cell Signaling Technologies), p-p38 MAPK (Cell Signaling Technologies), p38 MAPK (Cell Signaling Technologies), and HSP90 (Cell Signaling Technologies). Membranes were then incubated overnight at $4^{\circ} \mathrm{C}$. Appropriate secondary antibodies $(1: 2,000)$ were added for $1 \mathrm{~h}$ at room temperature and subsequently washed before visualization of the protein bands using enhanced chemiluminescence reagents (PerkinElmer) and quantified using Quantity One (Bio-Rad) software.

\section{Statistical Analyses}

Data are presented as mean \pm SEM where each individual donor was denoted by n. $P$ values were calculated by using unpaired Student's $t$-test or one-way ANOVA followed by Tukey's post-hoc test using Graphpad Prism 7 (Graphpad Software). P-values of $<0.05$ were deemed to be statistically significant.

\section{RESULTS}

\section{LPS Activation Increases Glycolysis in Human CD14 ${ }^{+}$CD16- Monocytes}

It is becoming increasingly appreciated that immune cells alter their metabolism when they become activated $(11,12)$. To determine the metabolic preference of human $\mathrm{CD} 14^{+} \mathrm{CD} 16^{-}$ monocytes after activation with LPS, we isolated cells from buffy coats of healthy volunteers via fluorescence-activated cell sorting (FACS) before placing them in the seahorse $\mathrm{XF}^{\mathrm{e}} 96$ bioanalyser to characterize their metabolic preference. We noted a significant increase in extracellular acidification rate (ECAR) within $20 \mathrm{~min}$ of LPS stimulation (Figures 1A,B) indicating increased rate of glycolysis. This was also associated with enhanced glucose uptake, measured via flow cytometry using 2NBDG (Figure 1C). Moreover, we found no significant change in oxygen consumption rate (OCR), which is a proxy of oxidative phosphorylation (OXPHOS), in $\mathrm{CD} 14^{+} \mathrm{CD} 16^{-}$monocytes after LPS stimulation (Figures 1D,E). We also used flow cytometry to quantify mitochondrial activity, which confirmed our findings from the seahorse bioanalyser (Figure 1F). These data suggest that stimulating monocytes with LPS causes an increase in glycolysis while not affecting OXPHOS.

\section{Inhibiting Glycolysis Decreases Monocyte Activation and Adhesion}

Given that we found $\mathrm{CD} 14^{+} \mathrm{CD} 16^{-}$monocytes increase glycolysis following LPS stimulation, we aimed to determine how important glucose utilization was to their ability to activate in response to LPS. To do this we used 2-Deoxy-Dglucose (2-DG), a glucose analog that enters into the cell like glucose but inhibits the first step of glycolysis via competitively blocking hexokinase, a rate limiting step of glycolysis (13). As expected, pre-treating cells with 2-DG reduced the increase in glycolysis caused by LPS (Figures 2A,B). Additionally, pretreating cells with 2-DG significantly inhibited the activation of the $\mathrm{CD} 14^{+} \mathrm{CD} 16^{-}$monocytes as determined by the cell surface 

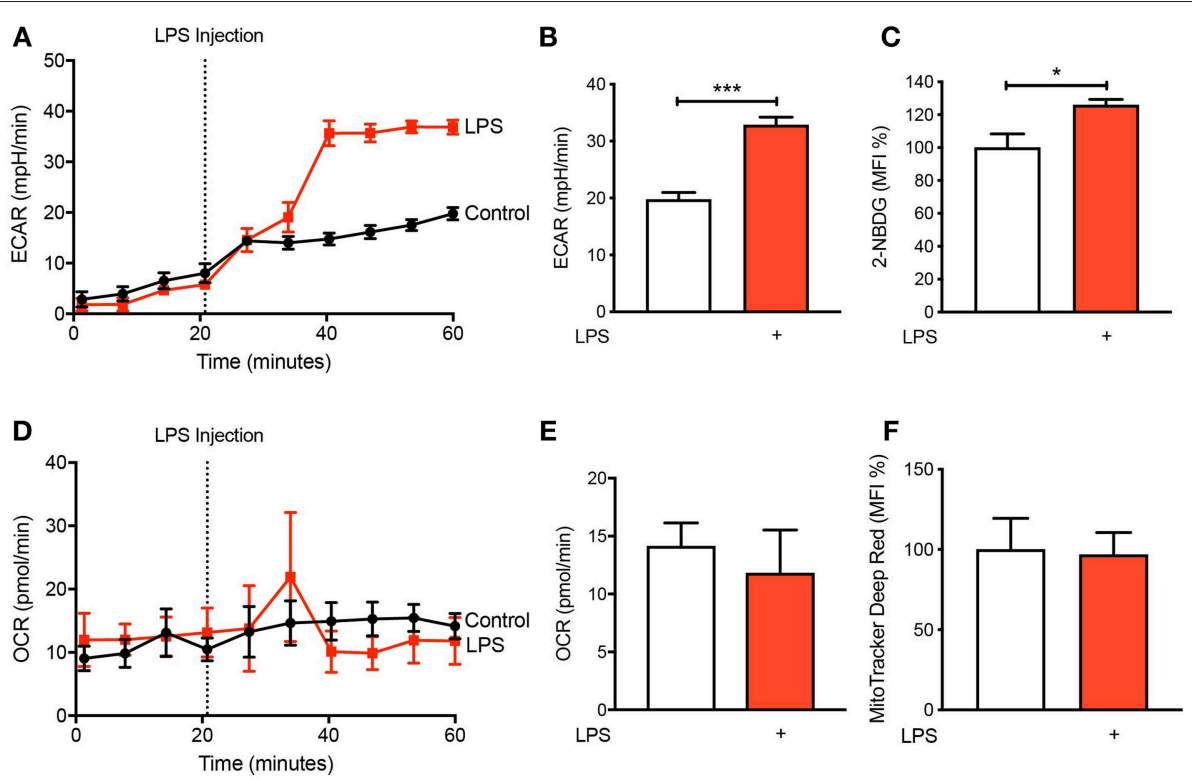

FIGURE 1 | LPS increases glycolysis in human CD14+ CD16 ${ }^{-}$monocytes. Isolated human CD14+ CD16 ${ }^{-}$monocytes were treated with or without 100 ng/ml LPS. A seahorse bioanalyser was used to measure extracellular acidification rate (ECAR) $(\mathbf{A}, \mathbf{B}) ; n=4$. Flow cytometry was used to measure glucose uptake using fluorescent analog 2-NBDG (C); $n=6$. A seahorse bioanalyser was used to measure oxygen consumption rate (OCR) $(\mathbf{D}, \mathbf{E}) ; n=4$. Mitochondrial activity was measured using flow cytometry $\mathbf{( F )} ; n=7$. Data are mean \pm SEM (un-paired $t$-test: ${ }^{\star} p<0.05,{ }^{\star \star \star} p<0.001$ ).

activation marker CD11b (Figure 2C). To functionally confirm the role of glycolysis in LPS-induced monocyte adhesion, we performed a static adhesion assay where we pre-coated wells with fibrinogen which allows activated monocytes to bind via CD11b. Monocyte adhesion was assessed using confocal microscopy where cells were also stained to quantify F-actin content as another measure of cell activation. As expected, following LPS stimulation, there was a significant increase in the number of monocytes adhering to fibrinogen as well as an increase in Factin content. These effects were blunted when cells were pretreated with 2-DG (Figures 2D-F). Furthermore, we performed a shear flow cell adhesion assay to monitor in real-time monocyte adhesion under shear stress in TNF- $\alpha$ activated mouse aorta ex vivo. We treated $\mathrm{CD} 14^{+} \mathrm{CD} 16^{-}$human monocytes with LPS $1 \mathrm{~h}$ before flowing the cells through the endothelium and found that there was a significant increase in monocyte adhesion. This increase in monocyte adhesion was abolished when pre-treating with 2-DG (Figures 2G-I). This confirms our hypothesis that blocking glycolysis prevents LPS-induced monocyte activation and adhesion.

\section{mTOR Is Involved in Regulating Glycolysis in LPS-Induced Monocytes}

To understand the mechanisms by which LPS increases glycolysis, we explored the mTOR pathway as it has been known to be involved in regulating glycolysis (14). Following LPS treatment we found a significant increase in the phosphorylation of $\mathrm{mTOR}$ in the $\mathrm{CD} 14^{+} \mathrm{CD} 16^{-}$monocytes and using rapamycin as a positive control for mTOR phosphorylation, phosphorylation of mTOR was significantly reduced as expected
(Figure 3A). The activation of mTOR is also known to further upregulate glucose transporter (GLUT)-1 to the cell surface in order to facilitate increased glucose uptake (15). When we measured GLUT-1 expression using flow cytometry, we found that LPS significantly increased cell surface GLUT-1 levels. When we inhibited mTOR activity using rapamycin, GLUT-1 expression was significantly blunted in LPS-induced monocytes (Figure 3B). This suggests that the increase in cell surface GLUT-1 is regulated by mTOR in monocytes. Furthermore, we also showed that inhibiting mTOR significantly prevented the increase in LPS-stimulated glycolysis (Figures 3C,D), suggesting mTOR is a master regulator of glycolysis in $\mathrm{CD} 14^{+} \mathrm{CD} 16^{-}$ monocytes. Next, we blocked mTOR to determine whether this affected the activation and adhesion of monocytes. When we pre-treated cells with rapamycin, we were able to prevent CD11b expression in LPS-induced monocytes (Figure 3E). The anti-inflammatory effects of mTOR blockade were also seen in the static adhesion assay where pre-treating cells with rapamycin prevented the number of monocytes adhered to fibrinogen. In addition, we found a decrease in F-actin content (Figures 3F-H). This suggests that the mTOR pathway is involved in LPSinduced activation and adhesion of $\mathrm{CD} 14^{+} \mathrm{CD} 16^{-}$monocytes by controlling glucose uptake and glycolysis.

\section{Blocking p-38 MAPK Signaling Prevents LPS-Induced Activation and Adhesion}

To further understand the signaling pathways mediating the activation and adhesion of LPS-stimulated monocytes, we measured mitogen-activated protein kinases (MAPKs), extracellular signal-related kinases (ERKs) and p38 MAPK, 

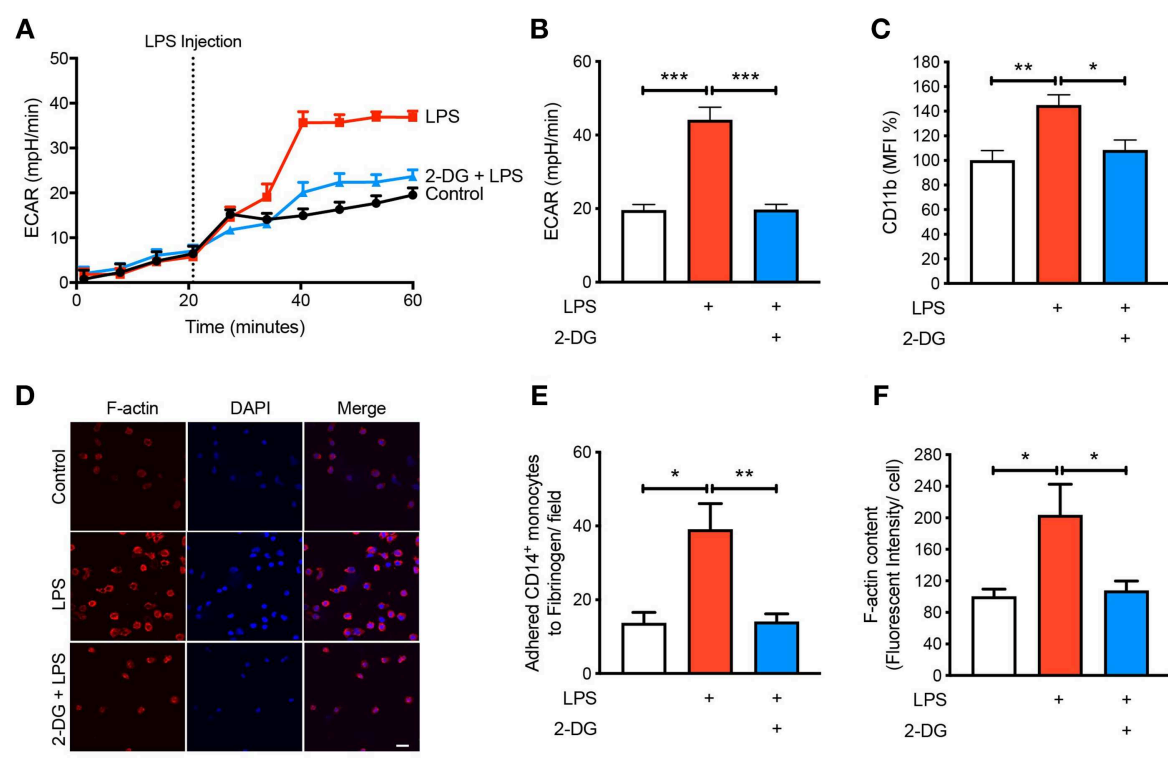

$\mathbf{F}$

G

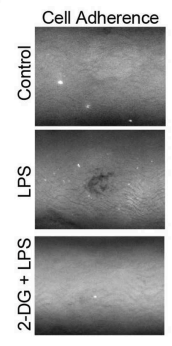

H

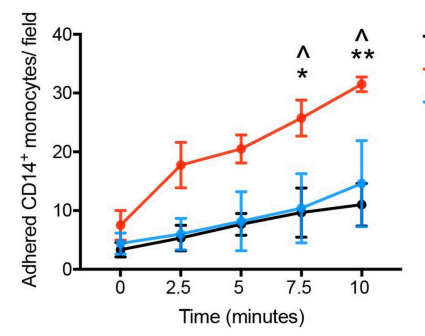

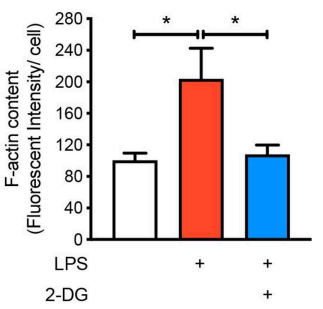

I

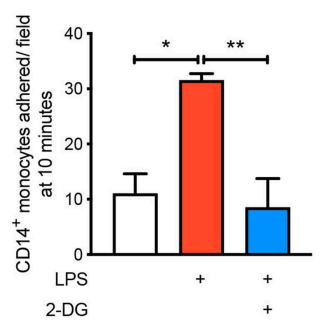

FIGURE 2 | Glycolysis is required for LPS-induced monocyte activation and adhesion. Isolated human CD14 ${ }^{+} \mathrm{CD}_{16}{ }^{-}$monocytes were pre-treated with or without $5 \mathrm{mM} 2-\mathrm{DG}$ for $1 \mathrm{~h}$ followed by $1 \mathrm{~h}$ of $100 \mathrm{ng} / \mathrm{ml}$ LPS stimulation. Seahorse bioanalyser was used to measure extracellular acidification rate (ECAR) (A,B); $n=4$. Flow cytometry was used to measure CD11b expression (C); $n=6-8$. Representative images (20 $\mu \mathrm{m}$ scale bar) (D) of the number of adhered monocytes (E) and F-actin content measured via confocal microscopy (F); $n=3-5$. Representative image of shear flow adhesion assay (white dots = adhered cells) (G). Quantification over 10 min time course $\mathbf{( H )}$ and at $10 \mathrm{~min}(\mathbf{I}) ; n=3-5$. Data are mean $\pm \mathrm{SEM}$ (one-way ANOVA with Tukey's test: $\left.{ }^{*} \& p<0.05,{ }^{\star \star} \& p<0.01,{ }^{\star \star \star} p<0.0001\right)$. In $\mathbf{( H )}$, denotes comparison between LPS vs. 2DG-LPS.

which are known to be activated during inflammation (16-18). As expected, both MAPKs were significantly phosphorylated when monocytes were treated with LPS (Figures 4A,B). Interestingly, blocking glycolysis using 2-DG did not prevent the phosphorylation of ERK1/2 (Figure 4A). Moreover, when we pre-treated cells with rapamycin and 2-DG, p38 MAPK phosphorylation was significantly decreased (Figure 4B). Therefore, we decided to inhibit p38 MAPK to determine whether this affected LPS-induced monocyte activation and adhesion. We employed SB-203580, an inhibitor of p38 MAPK, which confirmed that this pathway was involved in monocyte activation as we noted LPS was no longer able to increase the cell surface expression of CD11b (Figure 4C). Consistent with the inability of LPS to induce CD11b, we also found a reduction in the number of monocytes adhering to fibrinogen when p38 MAPK was inhibited (Figures 4D,E). To further confirm p38 MAPK occurs downstream of mTOR and glycolysis, we measured mTOR phosphorylation via western blot and also GLUT-1 levels via flow cytometry with SB-203580 in the presence of LPS and confirmed that phosphorylation of mTOR and GLUT-1 levels were unchanged (Figures 4F,G). These data suggest that LPS-mediated glycolysis and mTOR signaling induce p38 MAPK to promote $\mathrm{CD} 14^{+} \mathrm{CD} 16^{-}$monocyte activation and adhesion.

\section{Reactive Oxygen Species Are Involved in Glycolysis-Mediated Activation and Adhesion}

Next, we aimed to mechanistically link glycolysis with the induction of p38 MAPK signaling in driving $\mathrm{CD} 14^{+} \mathrm{CD} 16^{-}$ monocyte activation and adhesion. Inflammatory signaling can trigger p38 MAPK activation by ROS and preventing ROS accumulation using anti-oxidants averts p38 MAPK activation $(16,19,20)$. Since one of the by-products of glycolysis is ROS generation, we hypothesized that this may be the link between glycolysis and p38 MAPK activation. Indeed, we found that LPS increased ROS accumulation in monocytes via flow cytometry 


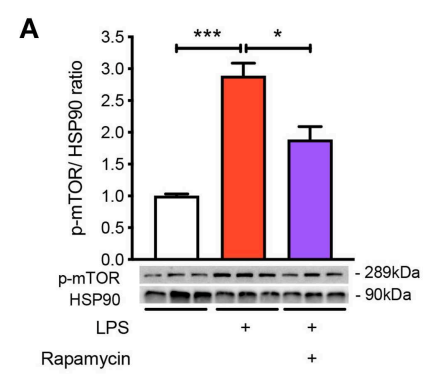

C

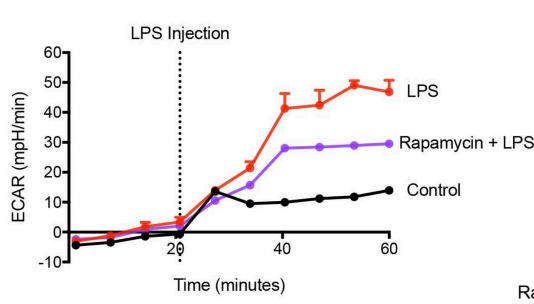

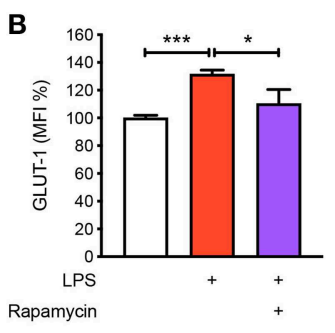

D

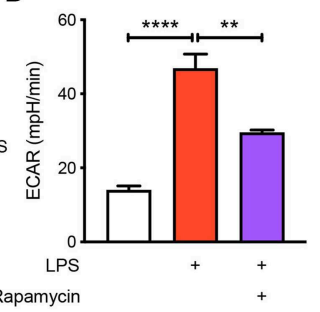

E

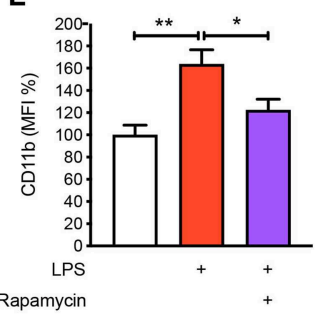

F

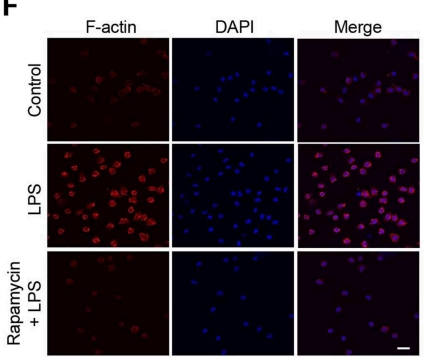

G

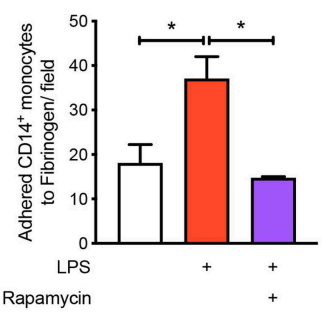

H

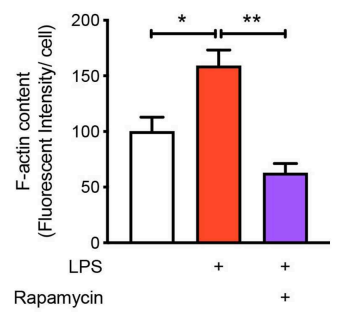

FIGURE 3 | mTOR pathway is involved in regulating glycolysis in LPS-induced monocytes. Isolated human CD14 ${ }^{+}$CD16 ${ }^{-}$monocytes were pre-treated with or without $20 \mathrm{nM}$ rapamycin an hour before $1 \mathrm{~h} 100 \mathrm{ng} / \mathrm{ml}$ LPS stimulation. $\mathrm{mTOR}$ phosphorylation was quantified by western blot at $30 \mathrm{~min}$ after LPS stimulation $(\mathbf{A})$; $n=$ 6-7. Flow cytometry was used to measure GLUT-1 expression; $n=3-5$ (B). Extracellular acidification rate (ECAR) was measured in real-time (C,D); $n=3-4$. CD11b expression was measured by flow cytometry (E); $n=6-7$. Static cell adhesion assay performed utilizing F-actin and DAPI stain via confocal microscopy (20 $\mu \mathrm{m}$ scale bar) (F-H); $n=3-4$. Data are mean \pm SEM (un-paired $t$-test and one-way ANOVA with Tukey's test: ${ }^{\star} p<0.05,{ }^{\star *} p<0.01,{ }^{\star \star *} p<0.001,{ }^{\star \star \star *} p<0.0001$ ).

(Figure 5A). Our data suggest that ROS generation was a consequence of increased glycolysis, as interventions upstream of glycolysis, rapamycin, and 2-DG, were able to block global ROS levels using the $\mathrm{H}_{2}$ DCFDA fluorescent indicator (Figure 5A). Moreover, to determine whether the increase in ROS production was generated from glycolysis and not glucose utilization via the mitochondria, we specifically stained for mitochondrial ROS using MitoSOX, which we found to be unchanged with LPS (Figure 5B). To confirm that mitochondrial ROS did not play a role during acute LPS activation, we treated cells with MitoQ that specifically reduces mitochondrial ROS and showed that global ROS levels (Figure 5C) and CD11b expression were unchanged (Figure 5D). We also measured ROS production in LPS-induced monocytes that were pre-treated with the p38 MAPK inhibitor SB-203580 and found no change in ROS, suggesting that p38 MAPK is downstream of ROS in LPS-activated monocytes (Figure 5E). Next, we inhibited ROS oxidation of cysteines using $\mathrm{N}$-acetyl-L-cysteine (NAC) and measured phosphorylation of p38 MAPK to delineate whether ROS activated p38 MAPK.
Indeed, we found that pre-treating cells with NAC before incubating with LPS prevented p38 MAPK activation but not ERK1/2 phosphorylation, suggesting that the ROS driven pathway was specific to p38 MAPK signaling (Figures 5F,G). These results show that ROS production in LPS-induced monocytes occurs downstream of the mTOR and glycolytic pathway but upstream of p38 MAPK. More importantly, inhibiting ROS production using NAC, significantly prevented LPS-induced CD11b expression (Figure 5H). Furthermore, NAC was able to affect LPS-induced monocyte adhesion as well as reduce F-actin formation (Figures $5 \mathbf{I}-\mathbf{K}$ ). These results indicate that LPS stimulation of monocytes triggers mTOR regulated glycolysis, which drives ROS, causing downstream p38 MAPK activation, resulting in monocyte activation and adhesion.

\section{DISCUSSION}

During an acute inflammatory response, monocytes become activated, adhere to the endothelium, and transmigrate into 

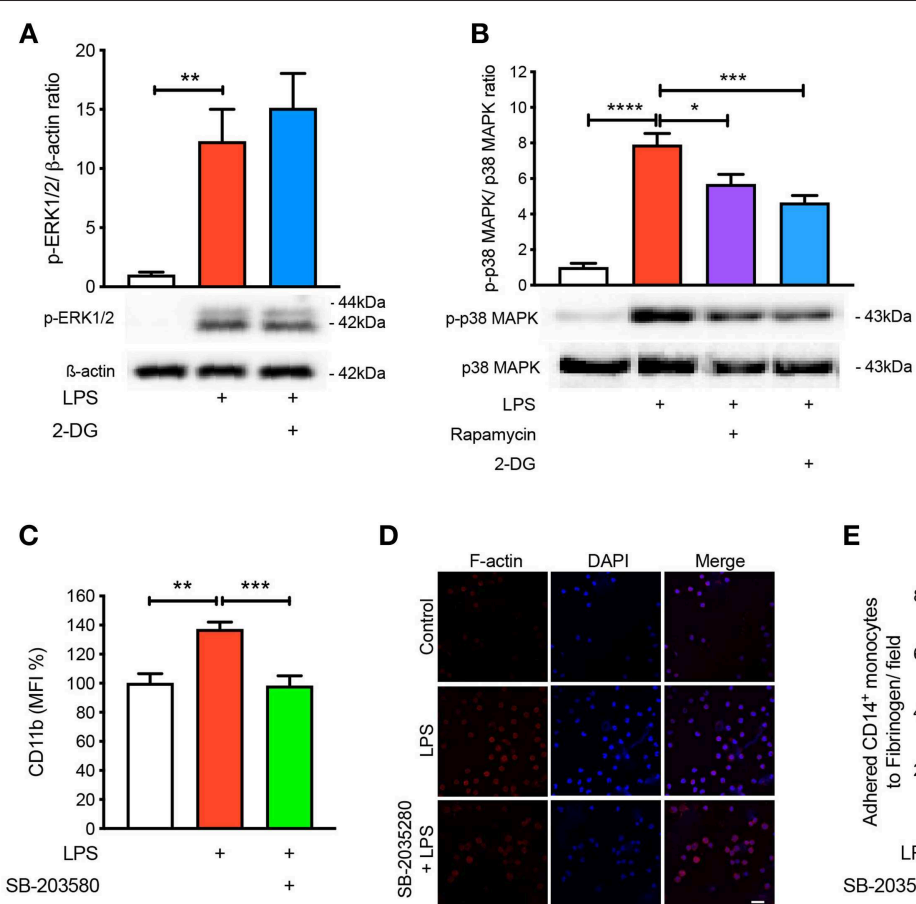

D

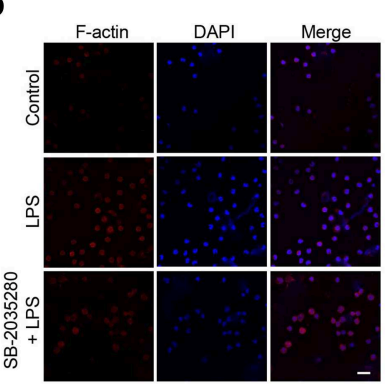

E

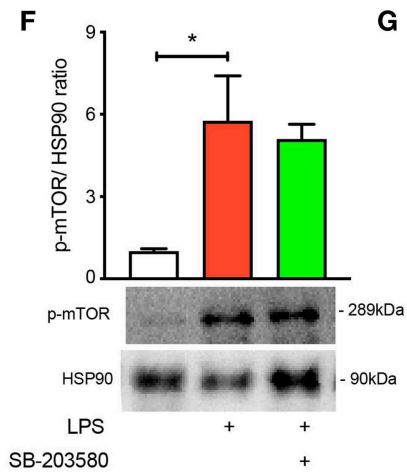

G

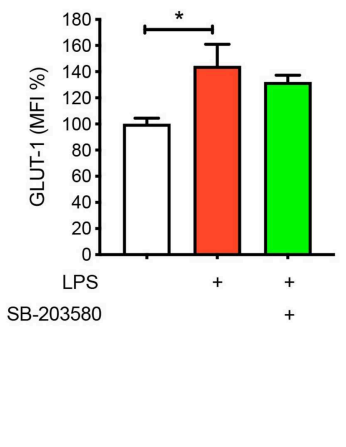

FIGURE 4 | p38 MAPK is involved in LPS-induced monocyte activation and adhesion. ERK1/2 phosphorylation were measured with or without 5 mM 2-DG followed by $1 \mathrm{~h}$ of $100 \mathrm{ng} / \mathrm{ml}$ LPS stimulation (A); $n=4-5$. p38 MAPK phosphorylation was measured with or without $5 \mathrm{mM} 2-\mathrm{DG}$ or $20 \mathrm{nM}$ rapamycin before $100 \mathrm{ng} / \mathrm{ml}$ LPS stimulation (B); $n=6-7$. Cells were pre-treated with $5 \mathrm{nM}$ SB-203580 followed by $1 \mathrm{~h}$ of $100 \mathrm{ng} / \mathrm{ml}$ LPS stimulation before measuring CD11b expression via flow cytometry (C); $n=8$. Static cell adhesion assay was performed utilizing F-actin and DAPI stain via confocal microscopy (20 $\mu \mathrm{m}$ scale bar); $n=3-4$ (D-E). mTOR phosphorylation was quantified by western blot at $30 \mathrm{~min}$ after LPS stimulation in the presence of $5 \mathrm{nM}$ SB-203580 (F); $n=3$. Flow cytometry was used to measure GLUT-1 expression; $n=3-4$ (G). Data are mean \pm SEM (one-way ANOVA with Tukey's test: ${ }^{*} p<0.05,{ }^{* *} p<0.01,{ }^{* \star *} p<0.001,{ }^{* * * *} p<0.0001$ ).

the inflamed tissue. However, the mechanistic pathways by which human $\mathrm{CD}_{14}{ }^{+} \mathrm{CD} 16^{-}$monocytes activate and adhere, in particular the specific metabolic pathways are yet to be fully elucidated. In this study, we revealed that increased flux through glycolysis is critical to induce the signaling pathways that monocytes rely on for adherence. We found that LPS-stimulated human $\mathrm{CD} 14^{+} \mathrm{CD} 16^{-}$monocytes increase CD11b expression and adhesion via the phosphorylation of mTOR which facilitates the uptake of glucose and glycolysis. When we further investigated the mechanistic link between glycolysis and adhesion, we found that an increase in glycolysis resulted in the production of ROS which activated the p38 MAPK pathway, leading to monocyte activation and adhesion.

Glycolysis and oxidative phosphorylation (OXPHOS) via the mitochondria are the two main metabolic pathways, which can control the overall phenotype of the cell. A classic example of this are the metabolic status of inflammatory and anti-inflammatory macrophages. Inflammatory or M1-like macrophages are highly glycolytic, while anti-inflammatory or M2-like macrophages have been found to be mitochondrial dependent, using both glucose and fatty acids for OXPHOS $(11,12)$. More importantly, when glycolysis or OXPHOS are inhibited using specific metabolic inhibitors it reduces the ability 

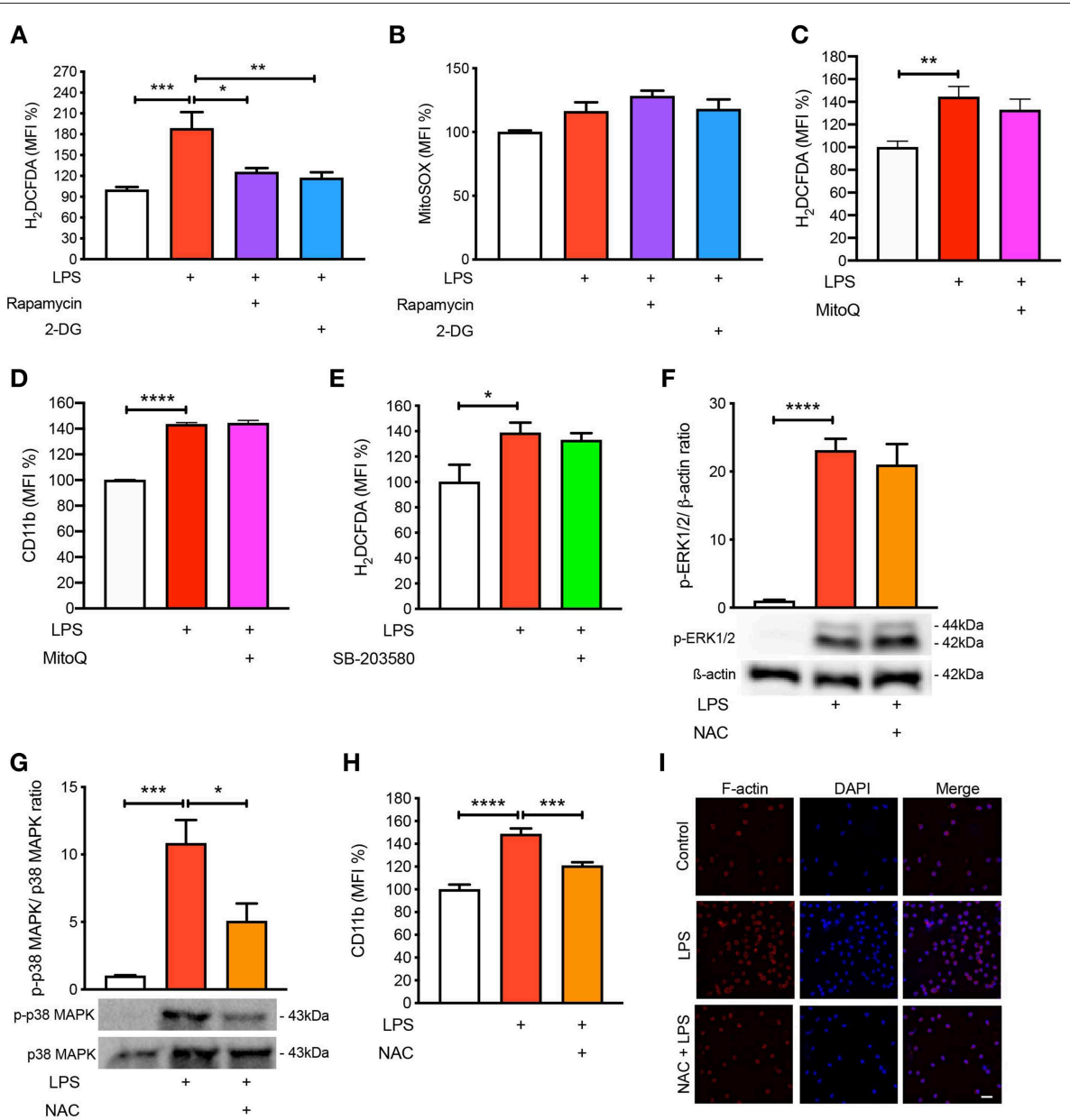

$\mathbf{I}$
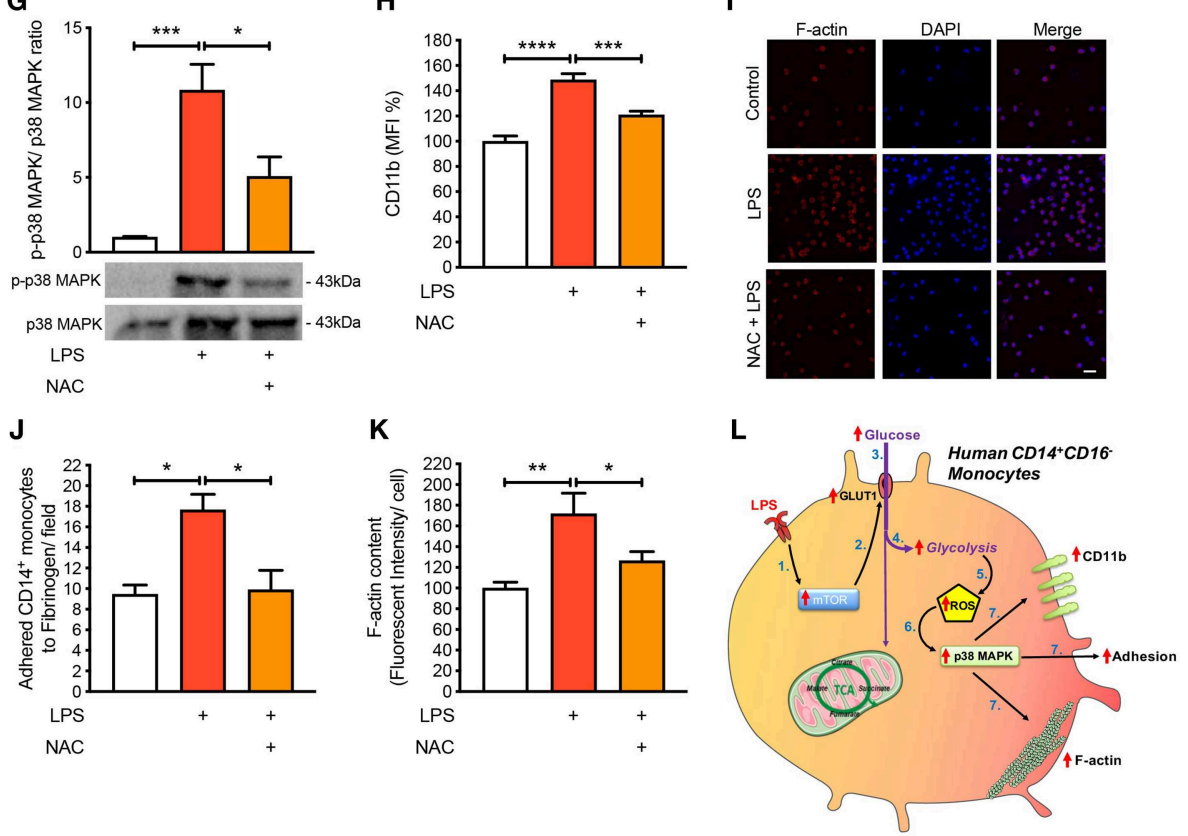

FIGURE 5 | ROS regulates p38 MAPK-induced monocyte activation and adhesion. Isolated human CD14+ ${ }^{+}$D16 ${ }^{-}$monocytes were pre-treated with or without $20 \mathrm{nM}$ rapamycin or $5 \mathrm{mM}$ 2-DG followed by $1 \mathrm{~h}$ of $100 \mathrm{ng} / \mathrm{ml}$ LPS stimulation before $\mathrm{H}_{2}$ DCFDA (A) and MitoSOX (B) levels were measured via flow cytometry; $n=4-8$. Cells were pre-treated with $100 \mathrm{nM}$ MitoQ followed by $1 \mathrm{~h}$ of $100 \mathrm{ng} / \mathrm{ml}$ LPS stimulation before $\mathrm{H}_{2}$ DCFDA (C) and CD11b (D) levels were measured via flow cytometry; $n=7-8$. Cells were pre-treated with $5 \mathrm{nM}$ SB-203580 followed by $1 \mathrm{~h}$ of $100 \mathrm{ng} / \mathrm{ml}$ LPS stimulation before measuring $\mathrm{H}_{2}$ DCFDA levels via flow cytometry (E); $n=4$. Cells were pre-treated with or without $1 \mathrm{mM} \mathrm{NAC}$ before stimulating with $100 \mathrm{ng} / \mathrm{ml}$ LPS for 30 min before measuring ERK1/2 (F) and p38 MAPK (G) phosphorylation; $n=4-5$. CD11b expression was measured via flow cytometry; $n=6-8 \mathbf{( H )} ; n=6-7$. Static cell adhesion assay performed utilizing F-actin and DAPI stain via confocal microscopy (20 $\mu \mathrm{m}$ scale bar) (I-K); $n=3-6$. Schematic diagram of proposed mechanistic pathway of acute LPS-induced CD14 ${ }^{+}$CD16 ${ }^{+}$ monocyte activation and adhesion (L). Data are mean \pm SEM (one-way ANOVA with Tukey's test: ${ }^{\star} p<0.05,{ }^{\star \star} p<0.01,{ }^{\star \star \star} p<0.001,{ }^{\star \star \star \star} p<0.0001$ ).

of macrophages to become inflammatory or anti-inflammatory, respectively. Here, we demonstrate a similar scenario in human $\mathrm{CD} 14^{+} \mathrm{CD} 16^{-}$monocytes where LPS increased glucose uptake and glycolysis. This is consistent with findings from Stienstra's group who also show an increase in glycolysis with LPS (21). However, it was somewhat surprising OXPHOS or mitochondrial activity was not reduced in the presence of LPS. This could suggest that the mitochondria does not play an essential role 
in providing the increase in energy metabolism for cellular activation in human $\mathrm{CD} 14^{+} \mathrm{CD} 16^{-}$monocytes during short $(1 \mathrm{~h})$ exposure compared to $24 \mathrm{~h}$ of LPS stimulation, where many other cellular changes are likely to be occurring (21). The non-reliance on the mitochondria in acute responses could be because $\mathrm{CD} 14^{+} \mathrm{CD} 16^{-}$monocytes do not require much energy as they are carried around the body by the circulatory system and require rapid activation upon an inflammatory stimulus and so have evolved to require little respirative metabolism. Thus, consistent with the hypothesis that $\mathrm{CD} 14^{+} \mathrm{CD} 16^{-}$monocytes use glycolytic metabolism, when this pathway was inhibited using 2-DG, we found a loss in the ability of these monocytes to increase cell surface CD11b and to adhere. This builds on a growing body of evidence that the cellular metabolic preference is a key determinate of cellular function.

Previously, it has been noted that mTOR is involved in regulating cell adhesion in cancer cells; however, the mechanistic pathway downstream of mTOR has not been explored. mTOR, the central regulator of cellular growth and proliferation, also governs glycolysis. Studies in BMDMs have shown that mTOR is responsible for upregulating glucose transporters, namely GLUT-1 (15). Glucose transporters are involved in facilitating the uptake of glucose which increases the rate of glycolysis. This pathway was also activated in our human $\mathrm{CD}_{14}{ }^{+} \mathrm{CD} 16^{-}$monocytes as inhibition of mTOR with rapamycin prevented GLUT-1 movement to the surface as well as glycolysis, leading to a reduction in monocytic activation and adhesion.

Delving further into the mechanisms linking glycolysis to the activation and adhesion of LPS-induced monocytes, LPS has previously been known to stimulate many activation pathways including p38 MAPK and ERK1/2 in human $\mathrm{CD} 14^{+} \mathrm{CD} 16^{-}$ monocytes. Additionally, p38 MAPK and ERK1/2 have been shown to regulate adhesion of tumor-associated macrophages, suggesting their involvement is important in cellular activation in order to cause adhesion to vessels or a matrix $(16,18)$. Interestingly, only p38 MAPK was found to be important in glycolysis-mediated cell adhesion in the $\mathrm{CD} 14^{+} \mathrm{CD} 16^{-}$ monocytes, suggesting that glycolytic events are key in regulating the phosphorylation of p38 MAPK. One of the stressors that is capable of signaling via p38 MAPK is ROS. Within the glycolytic pathway, we found that non-mitochondrial generated ROS appeared to activate p38 MAPK in order to cause LPSinduced monocyte activation. The source of non-mitochondrial ROS is likely to be NADPH-oxidase (NOX)-dependent, which is induced via the glycolytic pathway (22). Additionally, studies have shown that NOX enzymes are increased in human monocytes and macrophages upon inflammatory conditions in addition with increased ROS levels (23).

Clinical trials testing the benefits of antioxidants in CVD-related clinical trials have been disappointing, with many larger clinical trials showing no beneficial effects when given antioxidants such as vitamin $\mathrm{E}, \mathrm{C}$ or coenzyme $\mathrm{Q}$. After careful reflection on these trials, the limitations of these studies should be considered before closing the door to the therapeutic potential of antioxidants in CVD. These include dosage, efficacy, in vivo biological activity, specificity and statistical power (24-26). However, the importance of ROS in cellular metabolism in CVD is regaining traction. Importantly, we are now gaining a better understanding on the regulator of cellular ROS and how to harness the power of endogenous antioxidant pathways such as Nrf2 or restoring mitochondrial health (if the mitochondria is the driver) (27). Another important point to consider is the timing of intervention to therapeutic gain, perhaps antioxidants need to be administered earlier in life as opposed to testing this in middle to older aged participants in trial setting and formulating appropriate primary endpoints. How glycolysis regulates p38 MAPK is currently unknown, but given p38 MAPK is downstream of ROS, it is likely that $\mathrm{MKK} 3 / 6$, and potentially ASK1, are intermediary targets $(19,28)$. Nonetheless, LPS-induced glycolysis is required for the phosphorylation of p38 MAPK to cause monocyte activation.

In summary, we have found that the metabolism of glucose by human $\mathrm{CD} 14^{+} \mathrm{CD} 16^{-}$monocytes in response to LPS is critical for the activation of these cells (Figure 5L). These findings contribute to a larger body of evidence revealing that changes in cellular metabolism are central for the cell to respond to extrinsic stimuli (bacterial, viral, cytokines, etc). These metabolic changes assist the cell in performing effector functions and as such have become a key interest in disrupting unwanted processes, particularly in the immune system. Understanding these pathways and selectively inhibiting glycolysis may aid in chronic diseases where excessive monocyte recruitment is detrimental.

\section{DATA AVAILABILITY}

The datasets generated for this study are available on request to the corresponding author.

\section{AUTHOR CONTRIBUTIONS}

ML, AA-S, EC, WS, CB, OC, and MF conducted the experiments and analysis. ML, AA-S, AF, CP, GL, DH, $\mathrm{JH}$, and $\mathrm{AM}$ contributed to the experimental design and intellectual input. ML, AA-S, and AM wrote and revised the manuscript. All authors contributed to editing the manuscript.

\section{FUNDING}

This work was supported by NHMRC grant (APP1142398) to AM, GL, and JH. AM was supported by a Centenary Award from CSL. ML was supported by a postdoctoral fellowship from the National Heart foundation (101951). 


\section{REFERENCES}

1. Ley K, Laudanna C, Cybulsky MI, Nourshargh S. Getting to the site of inflammation: the leukocyte adhesion cascade updated. Nat Rev Immunol. (2007) 7:678-89. doi: 10.1038/nri2156

2. Liu L, Wei Y, Wei X. The immune function of Ly6Chi inflammatory monocytes during infection and inflammation. Curr Mol Med. (2017) 17:1-1. doi: 10.2174/1566524017666170220102732

3. Al-Sharea A, Lee MK, Whillas A, Michell D, Shihata W, Nicholls AJ, et al. Chronic sympathetic driven hypertension promotes atherosclerosis by enhancing hematopoiesis. Haematologica. (2018) 104:456-67. doi: 10.3324/haematol.2018.192898

4. Dragoljevic D, Kraakman MJ, Nagareddy PR, Ngo D, Shihata W, Kammoun HL, et al. Defective cholesterol metabolism in haematopoietic stem cells promotes monocyte-driven atherosclerosis in rheumatoid arthritis. Eur Heart J. (2018) 39:2158-67. doi: 10.1093/eurheartj/ehy119

5. Al-Sharea A, Lee M, Whillas A, Flynn MC, Chin-Dusting J, Murphy AJ. Nicotinic acetylcholine receptor alpha 7 stimulation dampens splenic myelopoiesis and inhibits atherogenesis in Apoe -/- mice. Atherosclerosis. (2017) 265:47-53. doi: 10.1016/j.atherosclerosis.2017.08.010

6. Fleetwood AJ, Lee M, Singleton W, Achuthan A, Lee M-C, O'BrienSimpson NM, et al. Metabolic remodeling, inflammasome activation, and pyroptosis in macrophages stimulated by Porphyromonas gingivalis and its outer membrane vesicles. Front Cell Infect Microbiol. (2017) 7:351. doi: 10.3389/fcimb.2017.00351

7. Shehata HM, Murphy AJ, kit Lee M, Gardiner CM, Crowe SM, Sanjabi S, et al. Sugar or fat?-metabolic requirements for immunity to viral infections. Front Immunol. (2017) 8:1311. doi: 10.3389/fimmu.2017.01311

8. Masson JJ, Murphy AJ, Lee MK, Ostrowski M, Crowe SM, Palmer CS. Assessment of metabolic and mitochondrial dynamics in CD4+ and CD8+ T cells in virologically suppressed HIV-positive individuals on combination antiretroviral therapy. PLOS ONE. (2017) 12:e0183931. doi: 10.1371/journal.pone.0183931

9. Tannahill G, Curtis A, Adamik J, Palsson-rmott E, McGettrick A, Goel G, et al. Succinate is an inflammatory signal that induces IL- $1 \beta$ through HIF- $1 \alpha$. Nature. (2013) 496:238-42. doi: 10.1038/nature11986

10. Cros J, Cagnard N, Woollard K, Patey N, Zhang S-Y, Senechal B, et al. Human CD14dim monocytes patrol and sense nucleic acids and viruses via TLR7 and TLR8 receptors. Immunity. (2010) 33:375-86. doi: 10.1016/j.immuni.2010.08.012

11. Huang S, Smith AM, Everts B, Colonna M, Pearce EL, Schilling JD, et al. Metabolic reprogramming mediated by the mTORC2-IRF4 signaling axis is essential for macrophage alternative activation. Immunity. (2016) 45:817-30. doi: 10.1016/j.immuni.2016.09.016

12. Huang S, Everts B, Ivanova Y, O'Sullivan D, Nascimento M, Smith A, et al. Cell-intrinsic lysosomal lipolysis is essential for alternative activation of macrophages. Nat Immunol. (2014) 15:846-55. doi: 10.1038/ni.2956

13. Aft R, Zhang F, Gius D. Evaluation of 2-deoxy-D-glucose as a chemotherapeutic agent: mechanism of cell death. Brit J Cancer. (2002) 87:805-12. doi: 10.1038/sj.bjc.6600547

14. Moon J-S, Hisata S, Park M-A, DeNicola GM, Ryter SW, Nakahira $\mathrm{K}$, et al. mTORC1-induced HK1-dependent glycolysis regulates NLRP3 inflammasome activation. Cell Rep. (2015) 12:102-15. doi: 10.1016/j.celrep.2015.05.046

15. Buller CL, Loberg RD, Fan M-H, Zhu Q, Park JL, Vesely E, et al. A GSK-3/TSC2/mTOR pathway regulates glucose uptake and GLUT1 glucose transporter expression. Am J Physiol Cell Physiol. (2008) 295:C836-43. doi: 10.1152/ajpcell.00554.2007

16. Li L, Leung DY, Goleva E. Activated p38 MAPK in peripheral blood monocytes of steroid resistant asthmatics. PLoS ONE. (2015) 10:e0141909. doi: 10.1371/journal.pone.0141909
17. Kim H, Ullevig SL, Zamora D, Lee C, Asmis R. Redox regulation of MAPK phosphatase 1 controls monocyte migration and macrophage recruitment. Proc National Acad Sci USA. (2012) 109:E2803-12. doi: 10.1073/pnas.1212596109

18. Kurosawa M, Numazawa S, Tani Y, Yoshida T. ERK signaling mediates the induction of inflammatory cytokines by bufalin in human monocytic cells. Am J Physiol Cell Physiol. (2000) 278:C500-8. doi: 10.1152/ajpcell.2000.278.3.C500

19. Noguchi T, Ishii K, Fukutomi H, Naguro I, Matsuzawa A, Takeda K, et al. Requirement of reactive oxygen species-dependent activation of ASK1-p38 MAPK pathway for extracellular ATP-induced apoptosis in macrophage. J Biol Chem. (2008) 283:7657-65. doi: 10.1074/jbc.M708402200

20. Son Y, Kim S, Chung H-T, Pae H-O. Methods in enzymology. Sect H2o2 Regul Cell Signal. (2013) 528:27-48. doi: 10.1016/B978-0-12-405881-1.00002-1

21. Lachmandas E, Boutens L, Ratter JM, Hijmans A, Hooiveld GJ, Joosten LA, et al. Microbial stimulation of different Toll-like receptor signalling pathways induces diverse metabolic programmes in human monocytes. Nat Microbiol. (2016) 2:16246. doi: 10.1038/nmicrobiol.2016.246

22. Azevedo EP, Rochael NC, Gulmaraes Costa AB, de Souza-Vieira TS, Ganilho J, Saraiva EM, et al. A metabolic shift toward pentose phosphate pathway is necessary for amyloid fibril- and phorbol 12-myristate 13-acetate-induce neutrophil extracellular trap (NET) formation. J Biol Chem. (2015) 36:2217483. doi: 10.1074/jbc.M115.640094

23. Manea A, Manea SA, Gan AM, Constantin A, Fenyo IM, Raicu M, et al. Human monocytes and macrophages express NADPH oxidase 5 ; a potential source of reactive oxygen species in atherosclerosis. Biochem Biophys Res Commun. (2015) 461:172-9. doi: 10.1016/j.bbrc.2015. 04.021

24. Manson JE, Gaziano JM, Spelsberg A, Ridker PM, Cook NR, Buring JE, et al. A secondary prevention trial of antioxidant vitamins and cardiovascular disease in women. Rationale, design, and methods. The WACS Research Group. Ann Epidemiol. (1995) 5:261-9. doi: 10.1016/1047-2797(94)0 0091-7

25. Stephens NG, Parsons A, Schofield PM, Kelly F, Cheeseman K, Mitchinson MJ. Randomised controlled trial of vitamin $\mathrm{E}$ in patients with coronary disease: Cambridge Heart Antioxidant Study (CHAOS). Lancet. (1996) 347:781-6. doi: 10.1016/S0140-6736(96)90866-1

26. Heart Protection Study Collaborative Group. MRC/BHF Heart protection study of antioxidant vitamin supplementation in 20,536 high-risk individuals: a randomised placebo-controlled trial. Lancet. (2002) 360:23-33. doi: 10.1016/S0140-6736(02)09328-5

27. Mills EL, Ryan DG, Prag HA, Dikovskaya D, Menon D, Zasiona Z, et al. Accumulation of succinate controls activation of adipose tissue thermogenesis. Nature. (2018) 556:133-7. doi: 10.1039/nature25986

28. Cuenda A, Rousseau S. p38 MAP-Kinases pathway regulation, function and role in human diseases. Biochimica Biophysica Acta. (2007) 1773:1358-75. doi: 10.1016/j.bbamcr.2007.03.010

Conflict of Interest Statement: The authors declare that the research was conducted in the absence of any commercial or financial relationships that could be construed as a potential conflict of interest.

The handling editor declared a past collaboration with the authors ML and AM.

Copyright (c) 2019 Lee, Al-Sharea, Shihata, Bertuzzo Veiga, Cooney, Fleetwood, Flynn, Claeson, Palmer, Lancaster, Henstridge, Hamilton and Murphy. This is an open-access article distributed under the terms of the Creative Commons Attribution License (CC BY). The use, distribution or reproduction in other forums is permitted, provided the original author(s) and the copyright owner(s) are credited and that the original publication in this journal is cited, in accordance with accepted academic practice. No use, distribution or reproduction is permitted which does not comply with these terms. 